\title{
6 MV Wedge Photon Beam Profiles with the Fricke Xylenol Gel Dosimeter
}

\author{
Lucas N. de Oliveira \\ Departamento de Física e Matemática, Faculdade de Filosofia, \\ Ciências e Letras de Ribeirão Preto, Universidade de São Paulo, \\ Av. Bandeirantes 3900, Monte Alegre, 14015-400, Ribeirão Preto, SP, Brazil and \\ Instituto Federal de Educação, Ciência e Tecnologia do Tocantins AE 310 Sul, \\ Avenida NS 10 esquina com a LO 05, Plano Diretor Sul, Palmas, TO, Brazil
}

\author{
Carmen Sandra Guzmán Calcina, Fernanda Cavalcante, and Adelaide de Almeida* \\ Departamento de Física e Matemática, Faculdade de Filosofia, \\ Ciências e Letras de Ribeirão Preto, Universidade de São Paulo, \\ Av. Bandeirantes 3900, Monte Alegre, 14015-400, Ribeirão Preto, SP, Brazil \\ Carlos Eduardo de Almeida \\ Laboratório de Ciências Radiológicas (LCR), Universidade do Estado de Rio de Janeiro, \\ Rua São Francisco Xavier, 524, Rio de Janeiro, RJ, Brazil
}

(Received on 24 June, 2008)

\begin{abstract}
Wedged beam are often used in clinical radiotherapy to compensate missing tissues and dose gradients. In this work, the Fricke Xylenol Gel (FXG) dosimeter was used for $6 \mathrm{MV}$ photons radiation wedge field profiles measurements, allowing to infer the wedge filter physical attenuation coefficient. This dosimeter is a chemical system of a Fe ${ }^{3+}$-Xylenol complex concentration, that when measured spectrophotometrically, the absorbance is directly proportional to the absorbed dose. From theses results one can infer that the FXG can be used also as an alternative dosimetric system for measurements of wedge filters.
\end{abstract}

Keywords: Fricke Xylenol Gel; physical filter, wedge beam profiles

\section{INTRODUCTION}

Radiotherapy is used for cancer treatment involving ionizing radiation to control malignant cells. For this reason, it is necessary not only to control the absorbed dose released to the target volume, but also in the normal tissues surrounding the tumor. For this purpose, the geometry related to the tumor and the type and energy of the ionizing radiation are necessary to obtain a dose distribution suitable for each treatment. In radiotherapy the dose homogeneity is one of the most important parameters required during the irradiation and it depends primarily on the patient contour and tissue heterogeneity. Physical and virtual wedge filters, with typical angles of $15^{\circ}, 30^{\circ} 45^{\circ}$ and $60^{\circ}$, are normally used to compensate that.

There are several papers reporting the results of wedge beam profile measurements from physical, virtual and dynamic filters, using different dosimeters such as diode $[1,2]$, ionization chamber [3-5], chemical dosimeter [6-8], film $[2,4]$ and numerical methods that use Monte Carlo calculation codes $[1,9]$. In this work the Fricke Xylenol Gel (FXG) was used to measure the beam profiles of physical wedge filters used in $6 \mathrm{MV}$ photons clinical beams.

The FXG has some interesting features such as, broad linear dependence with the absorbed dose from 0.5 up to 30 Gy for $\gamma$ and x-ray photons [10-13], atomic effective number of 7.75 and density of $1.050 \mathrm{~kg} / \mathrm{m}^{3}$, respectively near to 7.64 and $1.040 \mathrm{~kg} / \mathrm{m}^{3}$ for soft tissue [14]. This dosimeter is based in the $\mathrm{Fe}^{2+}$ to $\mathrm{Fe}^{3+}$ oxidation, forming a $\mathrm{Fe}^{3+}$-Xylenol complex, whose absorbance peak is centered in $585 \mathrm{~nm}$. All

${ }^{*}$ Electronic address: dalmeida@ffclrp.usp.br the absorbance measurements [15-17] were done with the visible spectrophotometric technique. The results are compared with the ones reported using Monte Carlo [1], film [4], ionization chamber [3, 4] and diodes [1]. Similar measurements, made with an ionization chamber of $0.016 \mathrm{~cm}^{3}$, are also reported here.

\section{MATERIALS AND METHODS}

The measurements were done using $10 \times 10,15 \times 15$ and $20 \times 20 \mathrm{~cm}^{2}$ radiation field sizes of $6 \mathrm{MV}$ photons beams, generated by a Siemens/Mevatron. Also wedge filters of $15^{\circ}, 30^{\circ}, 45^{\circ}$ and $60^{\circ}$, made of iron alloy with an effective atomic number of 25.93 and density of $7.8110^{3} \mathrm{~kg} / \mathrm{m}^{3}$ were used [18]. Typical absorbed doses of $2 \mathrm{~Gy}$ were delivered at $101.5,110$ and $120 \mathrm{~cm}$ source detector distances (SDD), for each combination of field sizes and wedge angles. The profiles were obtained for all wedge angles for the FXG samples and only with the PTW-Freiburg/TM31016-0120/AU604926 ionization chamber of $0.016 \mathrm{~cm}^{3}\left(\mathrm{IC}_{0.01}\right)$, for the $60^{0}$ wedge angle, the most stringent situation to corroborate the measurements done.

The FXG samples manufactured through the mixture of the concentrations of: $4 \%$ gelatin (300 Bloom-Aldrich), 0.1 $\mathrm{mM}$ xylenol orange, $25 \mathrm{mM}$ sulphuric acid, $0.5 \mathrm{mM}$ ferrous ammonium sulphate and 96\% Milli Q water [10, 11, 19, 20], were inserted in PMMA special cuvettes $\left(30 \times 30 \times 1 \mathrm{~cm}^{3}\right)$. An acrylic phantom, composed of a build-up plate of $30 \times$ $30 \times 1.5 \mathrm{~cm}^{3}$ and four plates of $30 \times 30 \times 2 \mathrm{~cm}^{3}$ was used in order to provide full backscatter condition to the measurements. The phantom was set on the machine table top, with the machine gantry at $0^{0}$, for both $\mathrm{FXG}$ and $\mathrm{IC}_{0.01}$ beam profiles measurements (Fig. 1). 


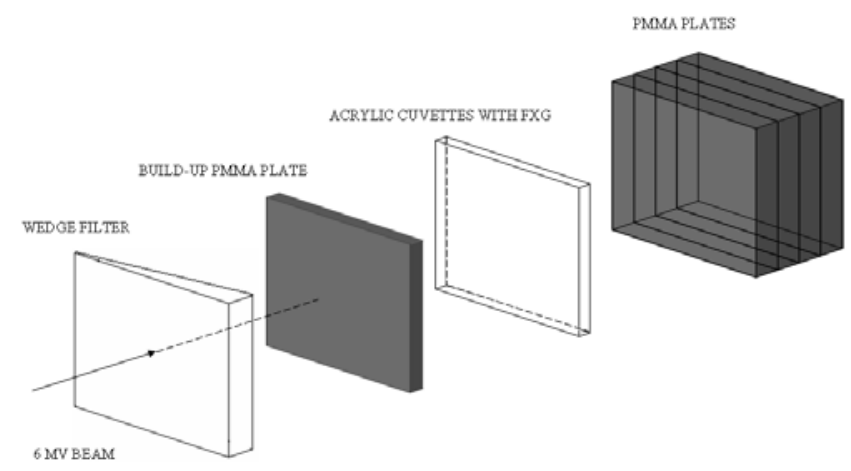

FIG. 1: Acrylic phantom for beam profile measurements, with four plates of $30 \times 30 \times 2 \mathrm{~cm}^{3}$ and a build-up plate with $1.5 \mathrm{~cm}$ thickness. The phantom, the cuvettes and the $\mathrm{IC}_{0.01}$ were irradiated perpendicularly to the beam.

The FXG absorbance measurements were obtained using a home made quasi monochromatic light intensity reader [13] (with a collimator aperture of $1 \mathrm{~mm}$ ). The system is composed of a light emission diode and a photodiode sensor, both with peaks near to $585 \mathrm{~nm}$, correspondent to the FXG maximum absorbance, previously determined in a visible spectrophotometer. For the irradiations, the FXG cuvettes were placed between the acrylic plates and the ionization chamber was inserted in the special acrylic plate of $\left(30 \times 30 \times 1 \mathrm{~cm}^{3}\right)$, to be placed between the other plates, like the FXG.

The natural oxidation of $\mathrm{Fe}^{+2}$ ions can be divided in natural and ionization radiation, the natural consideration aspects as: temperature variation, light intensity and influence with air [11, 21-23]. Both oxidations increases with the time, consequently the absorbance values also increase. In order to infer how the absorbance varieties with the time, six FXG samples were irradiated with $2 \mathrm{~Gy}$ of $6 \mathrm{MV}$ photons and were read immediately after irradiations $(t=0)$ and subsequent times till complety 5 hours. For the FXG system the absorbance behavior measurements (average for six values for each time value) is presented in Fig. 2 and a maximum uncertainty was calculated as $0.5 \%$ [24]. Together with these measurements, also the diffusion coefficient measurements were

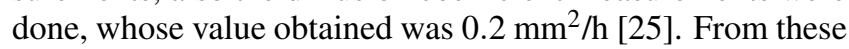
results one can infer that for up to 5 hours the absorbance values are almost time independent post-irradiation. With these results, we have decided to read the samples $30 \mathrm{~min}$ utes after irradiations. For the profiles measurements, three absorbed dose readings were done for each selected point, along the field size at depths of 1.5, 2.5, 9, 10, 20 and 21 $\mathrm{cm}$, respectively. The measured profile values were then normalized to the maximum value, at the field center. The normalized profiles were compared with data reported in the literature, Monte Carlo (MC) (DOSXYZ) [1], diode (area diameter $0.25 \mathrm{~cm}^{2}$ ) [1], X-OMAT V film (Kodak/Company) [4] and ionization chambers array (IC) (Wellhöfer /CA24) $[3,4]$. Although the literature values are for a $6 \mathrm{MV}$ photon beam, they were generated by different machines and in this way, different wedge delivery systems such as: Varian Physical Wedges [1], Siemens Virtual Wedge [3] and Varian Enhanced Dynamic Wedge [4], the comparisons are considered useful.

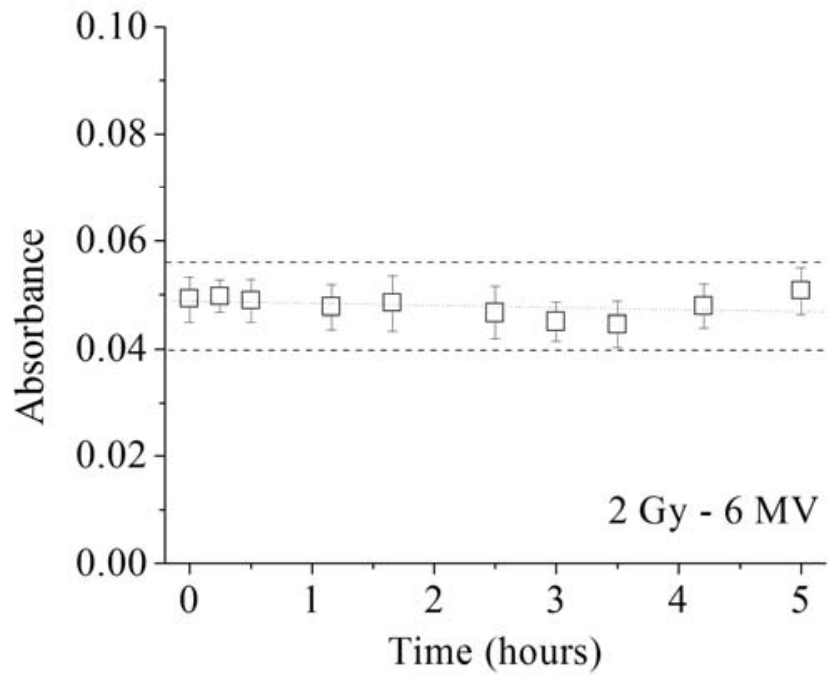

FIG. 2: FXG absorbance behavior versus time for samples irradiated with $2 \mathrm{~Gy}$ of a $6 \mathrm{MV}$ photon beam, $10 \times 10 \mathrm{~cm}^{2}$ field size and $101.5 \mathrm{~cm}$ SDD. These measurements show the absorbance independence with the time.

After the wedge filter profiles measurements, the effective linear attenuation coefficient of the physical filter $(\mu)$ could be derived from a selected point of the profile and correlated to the value at the center of the field, according to the following equation [26]:

$$
D(x, 1)=D(0,1) e^{-(\mu x \tan \alpha)}
$$

where, $D(0,1)$ and $D(x, 1)$ are the absorbed doses readings in/off the central axis respectively, $\mu$ is lateral distance (offaxis) and $\alpha$ is the angle provided by the wedge used. From all these values, (from the samples data) the attenuation coefficient can be inferred.

\section{RESULTS AND DISCUSSION}

In Fig. 3 the FXG and IC beam profile results for $60^{0}$ wedge filters are presented together with some data reported in the literature; for ionization chamber and film with $20 \times 20$ $\mathrm{cm}^{2}$ field size at depths of $1.5,10$ and $20 \mathrm{~cm}$. The dosimeters agreement for the $15^{0}, 30^{\circ}$ and $45^{\circ}$ wedge filters are very similar to those observed with $60^{\circ}$ wedge filters.

Table I presents the results from the FXG absorbed dose values compared with the values of ours ionization chamber and those reported values in the literature (IC [3, 4], MC [1], diode [1] and film [4]), considering the wedge filters angles, field sizes, measurement depths and $6 \mathrm{MV}$ photon beams.

From the literature [27, 28], the absolute percentage difference between two values can be derived from:

$$
\operatorname{diff} \%=\frac{\left(\mathrm{FXG}_{\text {value }}-\text { Dosimeters }_{\text {value }}\right)}{\mathrm{FXG}_{\text {value }}} \times 100
$$

The FXG values in this case, are taken as reference for the comparisons and using eq. (2), the average and maximum absolute percentage differences were obtained. 
TABLE I: Maximum differences in absorbed dose expressed in percentage among the FXG, $\mathrm{IC}_{0.01}$, MC, IC [3], IC [4], diode and film values. The small greek letters represent measurements depths as: $\alpha$ at $1.5 \mathrm{~cm}, \beta$ at $10 \mathrm{~cm}$ and $\gamma$ at $20 \mathrm{~cm}$, for $10 \times 10$ and $20 \times 20 \mathrm{~cm}^{2}$ field size. For the $15 \times 15 \mathrm{~cm}^{2}$ field size the depths were: $\alpha$ at $2.5 \mathrm{~cm}, \beta$ at $9 \mathrm{~cm}$ and $\gamma$ at $21 \mathrm{~cm}$.

\begin{tabular}{|c|c|c|c|c|c|c|c|c|c|c|c|c|}
\hline & & W15 & & & W30 & & & W45 & & & W60 & \\
\hline & $10 \times 10$ & $15 \times 15$ & $20 \times 20$ & $10 \times 10$ & $15 \times 15$ & $20 \times 20$ & $10 \times 10$ & $15 \times 15$ & $20 \times 20$ & $10 \times 10$ & $15 \times 15$ & $20 \times 20$ \\
\hline \multirow{4}{*}{$\mathrm{MC}[1]$} & - & $2.0^{\alpha}$ & - & - & $0.9^{\alpha}$ & - & - & $0.5^{\alpha}$ & - & - & $1.2^{\alpha}$ & - \\
\hline & - & $1.6^{\beta}$ & - & - & $1.3^{\beta}$ & - & - & $0.5^{\beta}$ & - & - & $0.7^{\beta}$ & - \\
\hline & - & $0.9^{\gamma}$ & - & - & $0.7^{\gamma}$ & - & - & $0.9^{\gamma}$ & - & - & $0.5^{\gamma}$ & - \\
\hline & $0.7^{\alpha}$ & - & $1.5^{\alpha}$ & $0.4^{\alpha}$ & - & $0.9^{\alpha}$ & $1.2^{\alpha}$ & - & $0.9^{\alpha}$ & $1.6^{\alpha}$ & - & $0.7^{\alpha}$ \\
\hline \multirow[t]{3}{*}{ IC[3] } & $1.2^{\beta}$ & - & $1.5^{\beta}$ & $0.8^{\beta}$ & - & $1.2^{\beta}$ & $1.9^{\beta}$ & - & $1.2^{\beta}$ & $1.4^{\beta}$ & - & $0.9^{\beta}$ \\
\hline & $1.2^{\gamma}$ & - & $1.6^{\gamma}$ & $1.4^{\gamma}$ & - & $0.8^{\gamma}$ & $1.6^{\gamma}$ & - & $1.2^{\gamma}$ & $1.1^{\gamma}$ & - & $1.1^{\gamma}$ \\
\hline & - & - & - & - & - & $0.5^{\alpha}$ & - & - & $1.7^{\alpha}$ & - & - & $0.6^{\alpha}$ \\
\hline \multirow[t]{3}{*}{$\mathrm{IC}[4]$} & - & - & - & - & - & $0.6^{\beta}$ & - & - & $1.1^{\beta}$ & - & - & $0.9^{\beta}$ \\
\hline & - & - & - & - & - & - & - & - & - & - & - & - \\
\hline & - & - & - & - & - & - & - & - & - & - & - & $0.4^{\alpha}$ \\
\hline \multirow[t]{3}{*}{$\mathrm{IC}_{0.01}$} & - & - & - & - & - & - & - & - & - & - & - & $0.8^{\beta}$ \\
\hline & - & - & - & - & - & - & - & - & - & - & - & $0.9^{\gamma}$ \\
\hline & - & $0.9^{\alpha}$ & - & - & - & - & - & $0.5^{\alpha}$ & - & - & $0.4^{\alpha}$ & - \\
\hline \multirow[t]{3}{*}{ Diode[1] } & - & $1.3^{\beta}$ & - & - & - & - & - & $0.8^{\beta}$ & - & - & $0.5^{\beta}$ & - \\
\hline & - & $1.5^{\gamma}$ & - & - & - & - & - & $0.8^{\gamma}$ & - & - & $0.7^{\gamma}$ & - \\
\hline & - & - & - & - & - & $0.6^{\alpha}$ & - & - & - & - & - & $0.5^{\alpha}$ \\
\hline \multirow[t]{2}{*}{ Film[4] } & - & - & - & - & - & $1.8^{\beta}$ & - & - & - & - & - & $1.5^{\beta}$ \\
\hline & - & - & - & - & - & - & - & - & - & - & - & - \\
\hline
\end{tabular}

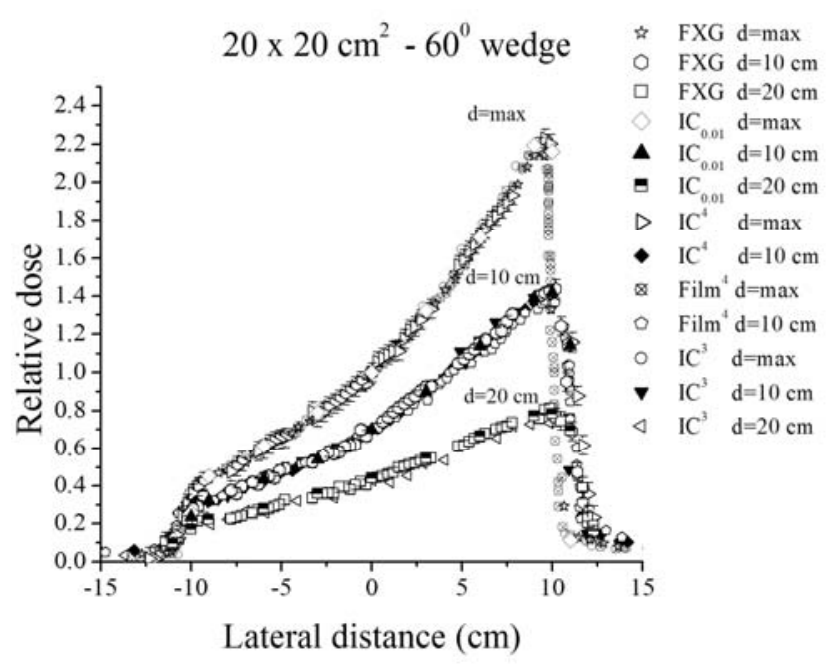

FIG. 3: Beam profile measurements comparison between FXG, $\mathrm{IC}_{0.01}$, IC and film for $6 \mathrm{MV}$ photon beams transmitted through a $60^{\circ}$ wedge physical filter and $20 \times 20 \mathrm{~cm}^{2}$ field size at the depths of $1.5,10$ and $20 \mathrm{~cm}$.

The maximum absolute percentage differences obtained are 2.0, 1.9, 1.8, 1.7, 1.5 and 0.9 , respectively for MC, IC [3], film, IC [4], diode [1] and $\mathrm{IC}_{0.01}$ and the average absolute percentage differences are 1.6, 1.2, 1.1, 0.9, 0.9, 0.7, respectively for $\mathrm{MC}$, film, IC [3], IC [4], diode and $\mathrm{IC}_{0.01}$. From these results, it can be inferred that the maximum differences compared to Monte Carlo [1], IC [3], film, IC [4], diode [1] and $\mathrm{IC}_{0.01}$ are smaller than $2 \%$. These differences can be related with the different machines spectral and wedge delivery systems.

From eq. (1), the effective linear attenuation coefficient may be calculated considering different off-axis distances and wedges angles. Using the eq. (1) and the $\mathrm{IC}_{0.01}$ and $\mathrm{FXG}$ data, the wedge filter linear attenuation coefficients, can be inferred as $0.0518 \pm 0.0001 \mathrm{~cm}^{-1}$ and $0.0513 \pm 0.0003 \mathrm{~cm}^{-1}$ respectively. These values are within $2.2 \%$ and $1.2 \%$ from $0.0507 \mathrm{~cm}^{-1}$ as reported by Santvoort [26].

\section{CONCLUSIONS}

We have presented the radiation beam profiles using physical wedge filters for a 6 MV Siemens Linac. The measurements were conducted for field sizes of $10 \times 10,15 \times 15$ and $20 \times 20 \mathrm{~cm}^{2}$, at several measurements depths. The results were compared to similar data reported in the literature, for different dosimeters (film, diode, ionization chamber array) and a Monte Carlo code. From these comparisons, one can infer that the FXG dose profile measurements are in close agreement, better than $1 \%$, with a very small ionization chamber and within $2.0 \%$ with the Monte Carlo code. Considering the effective linear attenuation coefficient for the Siemens Mevatron physical wedge filter, a difference of only $1.2 \%$ with the literature, could be attributed to the FXG results. According to all results obtained, it can be concluded that the dosimetric features of the FXG system clearly indicate its suitability for radiotherapy clinical beams measurements.

\section{ACKNOWLEDGMENTS}

The present work was supported by grants from CAPES (Brazil), CNPq (Brazil) and IAEA. 
[1] E. Spezi, D. G. Lewis, and C. W. Smith, "Monte Carlo simulation and dosimetric verification of radiotherapy beam modifiers", Physics in Medicine and Biology 46 (11), 3007-3029 (2001).

[2] D. D. Leavitt and L. Larsson, "Evaluation of a diode detector array for measurement of dynamic wedge dose distributions", Medical Physics 20, 381-382 (1993).

[3] M. Miften, X. R. Zhu, K. Takahashi, F. Lopez, and M. T. Gillin, "Implementation and verification of virtual wedge in a three-dimensional radiotherapy planning system", Medical Physics 27 (7), 1635-1643 (2000).

[4] H. H. Liu, E. P. Lief, and E. C. McCullough, "Measuring dose distributions for enhanced dynamic wedges using a multichamber detector array”, Medical Physics 24 (9), 1515-1519 (1997).

[5] A. M. Bidmead, A. J. Garton, and P. J. Childs, "Beam data measurements for dynamic wedges on Varian 600C (6 MV) and $2100 \mathrm{C}$ (6 and $10 \mathrm{MV}$ ) linear accelerators", Physics in Medicine and Biology 40 (3), 393-411 (1995).

[6] B. J. Tarte, P. A. Jardine, and T. van Doorn, "Laser-scanned agarose gel sections for radiation field mapping", International Journal of Radiation Oncology, Biology, Physics 36 (1), 175179 (1996).

[7] B. J. Tarte, P. A. Jardine, T. van Doorn, K. N. Nitschke, and M. G. Poulsen, "Development of a CCD array imaging system for measurement of dose distributions in doped agarose gels", Medical Physics 24 (9), 1521-1525 (1997).

[8] M. Bengtsson, T. Furre, J. Rodal, A. Skretting, and D. R. Olsen, "Measurement of dynamic wedge angles and beam profiles by means of MRI ferrous sulphate gel dosimetry", Physics in Medicine and Biology 41 (2), 269-277 (1996).

[9] F. Verhaegen and H. H. Liu, "Incorporating dynamic collimator motion in Monte Carlo simulations: an application in modelling a dynamic wedge", Physics in Medicine and Biology 46 (2), 287-296 (2001).

[10] M. A. Bero, W. B. Gilboy, P. M. Glover, and H. M. El-masri, "Tissue-equivalent gel for non-invasive spatial radiation dose measurements", Nuclear Instruments and Methods in Physics Research B 166-167, 820-825 (2000).

[11] A. M. Caldeira, A. M. Neto, A. C. Bento, M. L. Baesso, M. A. Silva, and A. de Almeida, "Behavior of oxidation in the radiochromic gel dosimeter through photoacoustic technique measurements", Applied Radiation and Isotopes 65 (5), 605609 (2007).

[12] R.T. Costa, "Radiotherapy Fricke Xylenol Gel dosimeter application", Universidade de So Paulo FFCLRP/DFM Master Thesis (2001).

[13] D.S. Felipe, "Development, test and application of a prototype reader for FXG dosimetry", Master Thesis - Universidade de So Paulo, FFCLRP/DFM (2003).

[14] F.M. Khan, The Physics of Radiation Therapy. (Williams \& Wilkins, Baltimore, 1984).

[15] C S Calcina, L N Oliveira, C E Almeida, and A Almeida, "Dosimetric parameters for small field sizes using Fricke xy- lenol gel, thermoluminescent and film dosimeters, and an ionization chamber", Physics in Medicine and Biology 52 (5), 1431-1439 (2007).

[16] G. Gambarini, G. Gomarasca, R. Marchesini, A. Pecci, L. Pirola, and S. Tomatis, "Three-dimensional determination of absorbed dose by spectrophotometric analysis of ferroussulphate agarose gel", Nuclear Instruments and Methods in Physics Research A 422, 643-648 (1999).

[17] G. Gambarini, M. Carrara, S. Gay, and S. Tomatis, "Dose imaging with gel-dosemeter layers: optical analysis and dedicated software," Radiation Protection Dosimetry 120 (1-4), 144-147 (2006).

[18] S. Rathee, C. B. Kwok, C. MacGillivray, and M. Mirzaei, "Commissioning, clinical implementation and quality assurance of Siemen's Virtual Wedge", Medical Dosimetry 24 (2), 145-153 (1999).

[19] L. J. Schereiner, "Review of Fricke gel dosimeters", Journal of Physics: Conference Series Third International Conference on Radiotherapy Gel Dosimetry 3, 9-21 (2004).

[20] M.V. Moreira, A. Almeida, R.T. Costa, and L.A. Perles, "FXG mass attenuation coefficient evaluation for radiotherapy routine", Journal of Physics - D (Applied Physics) 3, 146-149 (2004).

[21] B. L. Gupta and G. R. Narayan, " $\mathrm{G}\left(\mathrm{Fe}^{+3}\right)$ values in the FBX dosimeter", Physics in Medicine and Biology 30 (4), 337-340 (1985).

[22] S. Ohno, K. Furukawa, M. Taguchi, T. Kojima, and H. Watanabe, "Predicted radiolysis yield in a Fricke solution irradiated with various heavy ions", Radiat Phys Chem (1999).

[23] N. N. Bhat, D. Choudhary, A. Sarma, B. L. Gupta, and K. Siddappa, "Response of an FBX dosimeter to high LET ${ }^{7} \mathrm{Li}$ and ${ }^{12}$ C ons", Radiation Protection Dosimetry 61 (3), 433-435 (2003).

[24] IAEA (International Atomic Energy Agency) TRS-398, Absorbed Dose Determination in External Beam Radiotherapy: An International Code of Practice for Dosimetry based on Standards of Absorbed Dose to Water. (2004).

[25] L. N. de Oliveira, R. L. Zimmerman, M. V. Moreira, L.F. Pirani, D. Ila, A. de Almeida, "Determination of Diffusion Coefficient in Fricke Xylenol Gel Dosimeter after Electron Beam Bombardment" Proceeding of the International Conference on Surface Modification of Materials by Ion Beams - SMMIB 2007.

[26] J. van Santvoort, "Dosimetric evaluation of the Siemens Virtual Wedge", Physics in Medicine and Biology 43 (9), 26512663 (1998).

[27] V. Y. Kuperman, "A new analytical model for Varian enhanced dynamic wedge factors", Physics in Medicine and Biology 49 (13), 2841-2851 (2004).

[28] L. Chang, S. Y. Ho, and H. H. Chen, "Modelling the wedge shape for the virtual wedge", Physics in Medicine and Biology 48 (12), 1785-1794 (2003). 